\title{
Cross-species prophylactic efficacy of Sm-p80-based vaccine and intracellular localization of Sm-p80/Sm-p80 ortholog proteins during development in Schistosoma mansoni, Schistosoma japonicum, and Schistosoma haematobium
}

\author{
Adebayo J. Molehin ${ }^{1,2}$ • Souad R. Sennoune ${ }^{1,2}$ • Weidong Zhang ${ }^{1,2}$ • Juan U. Rojo ${ }^{3}$. \\ Arif J. Siddiqui $^{1,2}$ - Karlie A. Herrera ${ }^{1,2}$ - Laura Johnson ${ }^{1,2}$ • Justin Sudduth ${ }^{1,2}$. \\ Jordan May $^{1,2}$ - Afzal A. Siddiqui ${ }^{1,2}$
}

Received: 29 August 2017 / Accepted: 25 September 2017 /Published online: 12 October 2017

(C) Springer-Verlag GmbH Germany 2017

\begin{abstract}
Schistosomiasis remains a major global health problem. Despite large-scale schistosomiasis control efforts, clear limitations such as possible emergence of drug resistance and reinfection rates highlight the need for an effective schistosomiasis vaccine. Schistosoma mansoni large subunit of calpain (Sm-p80)-based vaccine formulations have shown remarkable efficacy in protecting against $S$. mansoni challenge infections in mice and baboons. In this study, we evaluated the cross-species protective efficacy of Sm-p80 vaccine against $S$. japonicum and $S$. haematobium challenge infections in rodent models. We also elucidated the expression of Sm-p80 and Sm-p80 ortholog proteins in different developmental stages of S. mansoni, S. haematobium, and S. japonicum. Immunization with Sm-p 80 vaccine reduced worm burden by $46.75 \%$ against $S$. japonicum challenge infection in mice. DNA prime/protein boost $(1+1$ dose administered on a single day) resulted in $26.95 \%$ reduction in worm burden in
\end{abstract}

Adebayo J. Molehin and Souad R. Sennoune contributed equally to this work and should be considered as equal first authors.

Electronic supplementary material The online version of this article (https://doi.org/10.1007/s00436-017-5634-4) contains supplementary material, which is available to authorized users.

Afzal A. Siddiqui

afzal.siddiqui@ttuhsc.edu

1 Center for Tropical Medicine and Infectious Diseases, School of Medicine, Texas Tech University Health Sciences Center, Lubbock, TX, USA

2 Department of Internal Medicine, School of Medicine, Texas Tech University Health Sciences Center, Lubbock, TX, USA

3 College of Life Sciences and Agriculture, University of New Hampshire, Durham, NH, USA
S. haematobium-hamster infection/challenge model. A balanced Th1 (IFN- $\gamma$, TNF- $\alpha$, IL-2, and IL-12) and Th2 (IL-4, IgG1) type of responses were observed following vaccination in both $S$. japonicum and S. haematobium challenge trials and these are associated with the prophylactic efficacy of Sm-p80 vaccine. Immunohistochemistry demonstrated that Sm-p80/ Sm-p80 ortholog proteins are expressed in different life cycle stages of the three major human species of schistosomes studied. The data presented in this study reinforce the potential of Sm-p80-based vaccine for both hepatic/intestinal and urogenital schistosomiasis occurring in different geographical areas of the world. Differential expression of Sm-p80/Sm-p80 protein orthologs in different life cycle makes this vaccine potentially useful in targeting different levels of infection, disease, and transmission.

Keywords Schistosomiasis · Cross-species protection · Sm-p80 vaccine $\cdot$ Sm-p80 immunolocalization

\section{Introduction}

Schistosomiasis, a chronic disease caused by parasitic helminths of the genus Schistosoma, remains a global public health problem. Over 240 million people are estimated to be currently infected worldwide with the majority being schoolaged children (Barry et al. 2013; Molehin et al. 2016). Experts in the field believe that the current estimate of the burden of schistosomiasis is most likely between 400 and 600 million (Hotez et al. 2014). Depending on reporting sources, the total disability-adjusted life years (DALYs) attributable to schistosomiasis range between 3.3 million to 36 million DALYs (ElKhoby et al. 2000; van der Werf et al. 2003). The mainstay for 
the control of schistosomiasis is mass drug administration (MDA) of praziquantel (PZQ) through MDA control programs (Gryseels et al. 2006). However, the outcomes of these control programs have been largely suboptimal due to insufficient coverage and lack of sustained reduction in the prevalence and transmission of the disease (El Ridi et al. 2015; Fonseca et al. 2015). Coverage data indicate that less than satisfactory numbers of schistosomiasis-affected people are being treated with PZQ, highlighting the shortcomings of the current control program (Hotez 2009; Ricciardi et al. 2016). In addition, these MDA programs would require optimum and continuous distribution of PZQ in endemic areas for sustainable disease control. Therefore, an effective vaccination strategy integrated with current treatment interventions presents a judicious approach for sustainable control and possible elimination of schistosomiasis. Progress towards elimination of schistosomiasis would only be made through an integrated approach which combines current control measures, such as MDA, health education, improved sanitation, and intermediate host control with an effective schistosomiasis vaccine (Mo et al. 2014).

The discovery and subsequent functional characterization of Schistosoma mansoni Sm-p80 as one of the key proteins involved in schistosome membrane biogenesis and/or renewal have provided an excellent target for a schistosome vaccine (Andresen et al. 1991; Siddiqui et al. 1993). Sm-p80 is expressed in intra-mammalian stages of the parasite and has been localized to the parasite surface syncytium making it an attractive target of host immunity (Braschi and Wilson 2006; Kumagai et al. 2005). With respect to the schistosomiasis vaccine development, Sm-p80 is now considered one of the leading candidates (Mo et al. 2014; Molehin et al. 2016). Several studies by our group have shown that Sm-p80 vaccine is highly efficacious against $S$. mansoni infections in mice and baboons (Ahmad et al. 2009a, b, c; Karmakar et al. 2014b; Le et al. 2014). Of equal importance is the finding from our recent study which showed that Sm-p80 vaccine formulated in glucopyranosyl lipid adjuvant-stable emulsion (GLA-SE) conferred cross-species prophylactic protection against $S$. haematobium infections in both hamster and baboon models (Karmakar et al. 2014a). GLA-SE is a toll-like receptor 4 (TLR4) agonist and a potent stimulator of antigen presenting cells currently being used in many commercially available vaccine formulations (Coler et al. 2011; Garcon and Van Mechelen 2011).

Based on our findings on the cross-species protective efficacy of Sm-p80 vaccine formulated in GLA-SE with the S. haematobium model, we hypothesized that Sm-p80-based induction of host immune responses would provide an opportunity to control Asiatic schistosomiasis caused by S. japonicum infections. In this present study, we evaluated the cross-species prophylactic efficacy of $\mathrm{Sm}-\mathrm{p} 80$ vaccine formulated in GLA-SE against $S$. japonicum infections in mice.
Most of the vaccination strategies employed by our group utilized three vaccine formulations at monthly intervals to animals. However, more recently, we demonstrated that simultaneous priming of mice with Sm-p80 DNA vaccine and boosting with recombinant Sm-p80 protein (DNA prime/ protein boost) on a single day with no further vaccine inoculations protected immunized mice against challenge infection with $S$. mansoni cercariae (Le et al. 2014). In this study, we also explored the cross-species protective efficacy of Sm-p80 vaccine (DNA prime/boost inoculation) in vaccinated hamsters against $S$. haematobium infection. An advantage of this prime-boost approach (if successful) is the benefit it provides in field conditions where compliance issues may arise if multiple vaccine boosters are required to achieve optimal vaccine efficacy. Further argument to support our hypothesis on the Sm-p80 vaccine-induced cross-species protection was also based on the expression profile of Sm-p80 ortholog protein in the different life cycle stages of $S$. japonicum and S. haematobium parasites. Schistosoma mansoni calpain (Uniprot ID: P27730) share 95 and 84\% amino acid sequence homology with $S$. haematobium calpain (Uniprot ID: A5A6F7) and $S$. japonicum calpain (Uniprot ID: O96072), respectively. We believe that a schistosome vaccine offering protection against multiple species of schistosomes and thereby providing relief from the clinical manifestations associated with hepatic/intestinal and urogenital schistosomiasis will be greatly beneficial in reducing the global burden of the disease in all of the major geographical areas where different parasite species-induced diseases are endemic.

\section{Methods}

\section{Animals, parasites, and ethic statement}

Female C57BL/6 mice (3-4 weeks old) were purchased from Charles River Laboratories (Wilmington, MA, USA). Schistosoma japonicum (Chinese strain)-infected Oncomelania hupensis and Schistosoma haematobium-infected Bulinus truncatus snails were procured from the Schistosome Resources Center (Biomedical Research Institute, Rockville, MD, USA). All animal procedures were conducted in accordance with Institutional Animal Care and Use Committee (IACUC) Guidelines (Protocol Number 20010202) and were approved by the Animal Ethics Committee at Texas Tech University Health Sciences Center.

\section{Immunofluorescence}

Cercariae and/or schistosomulae were fixed in $4 \%$ paraformaldehyde for $30 \mathrm{~min}$. Fixed specimens were allowed to settle under gravity and were washed in PBS three times and incubated in $50 \mathrm{mM} \mathrm{NH}{ }_{4} \mathrm{Cl}$ in PBS. Fixed samples were then 
blocked in a blocking solution (BSA 5\%, Triton X-100 0.02\% in PBS) for $3 \mathrm{~h}$ at room temperature. The samples were then incubated overnight at $4{ }^{\circ} \mathrm{C}$ with either rabbit anti-Sm-p 80 polyclonal antibodies (Fabgennix, USA) or Sm-p80 antiserum from mice or baboon diluted $1 / 300$ in $1 \%$ BSA in PBS. Following several washes in $1 \%$ BSA in PBS, the samples were incubated either in goat anti-rabbit or anti-human or anti-mouse Alexa Fluor highly cross-absorbed 647 (Molecular Probes, Thermo Fisher Scientific) for at least $2 \mathrm{~h}$ at $4{ }^{\circ} \mathrm{C}$. Samples were washed several times in PBS and mounted with Prolong gold antifade reagent with DAPI (Molecular Probes, Thermo Fisher Scientific). The schistosome eggs were processed as described above with an additional step. The eggs were treated with $1 \%$ SDS in PBS for $1 \mathrm{~h}$ at room temperature after being fixed and washed with PBS. The adult worms were fixed for $30 \mathrm{~min}$ at room temperature in $4 \%$ formaldehyde in PBS, followed by several washes in PBS. Samples were incubated twice in $50 \mathrm{mM} \mathrm{NH}_{4} \mathrm{Cl}$ in PBS ( 5 min each) followed by $1 \%$ BSA for 5 min twice and then for at least $3 \mathrm{~h}$ in $5 \% \mathrm{BSA}+0.02 \%$ Triton X-100 in PBS. The samples were incubated overnight at $4{ }^{\circ} \mathrm{C}$ with the primary antibody Sm-p80 diluted 1/300 in 1\% BSA. Following several washes in $1 \%$ BSA in PBS, the samples were incubated for $10 \mathrm{~min}$ in 5\% BSA at $4{ }^{\circ} \mathrm{C}$ before incubating with the secondary antibody, Alexa Fluor highly cross-absorbed 647 (Molecular Probes, Thermo Fisher Scientific) for $2 \mathrm{~h}$ at $4{ }^{\circ} \mathrm{C}$. Samples were washed several times in PBS and mounted with Prolong gold antifade reagent with DAPI (Molecular Probes, Thermo Fisher Scientific). All of the samples were visualized using a Nikon T1-E microscope with A1 confocal, and images were taken and analyzed using NIS software.

\section{Cloning, expression, and purification of recombinant Sm-p80}

Recombinant Sm-p80 (rSm-p80) was produced using prokaryotic expression system. Briefly, the full-length coding sequence of $S m-p 80$ gene sequence (GenBank accession number M74233) was cloned into pCold II vector (GenScript Corp., Piscataway, NJ, USA). The recombinant plasmid was transformed into BL21 (DE3) Escherichia coli strain. Production of recombinant protein was induced with $0.75 \mathrm{mM}$ isopropyl $\beta$-D-1-thiogalactopyranoside (IPTG) at mid-log phase $\left(\mathrm{OD}_{600} \sim 0.6-0.8\right)$ at $15^{\circ} \mathrm{C}$ and bacterial cells were harvested $24 \mathrm{~h}$ post-induction. Harvested cells were lysed with denaturing lysis buffer $\left(49.9 \mathrm{mM} \mathrm{NaH}_{2} \mathrm{PO}_{4}\right.$, $299 \mathrm{mM} \mathrm{NaCl}, 8 \mathrm{M}$ Urea, and $19 \mathrm{mM}$ Imidazole, $\mathrm{pH}$ 8.0) followed by sonication. Cell lysate was then centrifuged at $18,000 \times g$ for $30 \mathrm{~min}$ at $4{ }^{\circ} \mathrm{C}$. The supernatant was collected and centrifuged again as above to remove remaining cellular debris. The expressed protein was purified by utilizing immobilized metal affinity chromatography (IMAC) (Bio$\mathrm{Rad}, \mathrm{CA}, \mathrm{USA}$ ) followed by size exclusion chromatography using Sephadex G-150 columns. The purified denatured protein was refolded through step-wise dialysis in several exchanges of dialysis buffer (20 mM Tris, $150 \mathrm{mM} \mathrm{NaCl}, 5 \%$ Glycerol, $\mathrm{pH}-8.0$ ) and the protein concentrated using Spin-X UF concentrator (Corning, MA, USA). Endotoxin levels in protein samples were analyzed with a Limulus amebocyte lysate assay (Charles River Laboratories International, Inc., Wilmington, MA, USA). The quality of recombinant Smp80 produced was analyzed by sodium dodecyl sulfate polyacrylamide gel (SDS-PAGE) and Western blotting using horseradish peroxidase (HRP)-conjugated antibody directed against the recombinant His-tag (Thermo Fisher Scientific, MA, USA) (Online Resource 1). Endotoxin levels in the recombinant Sm-p80 used in immunizations were below that approved by the Food and Drug Administration for human use (approximately $0.06 \mathrm{EU} / \mathrm{ml}$ ).

\section{Preparation of Sm-p80 DNA vaccine}

DNA vaccine was prepared as previously described (Ahmad et al. 2009a). In brief, full-length coding sequence of $S m-p 80$ gene was cloned into BamHI/BglII sites VR1020 vector (VICAL Incorporated, CA, USA). The recombinant VR1020/Sm-p80 plasmid DNA was isolated via conventional alkaline lysis method and subsequently purified on Sepharose CL4B columns. Purified recombinant DNA vaccine was analyzed by restriction digestion and gel electrophoresis (Online Resource 1).

\section{Immunization protocol}

GLA-SE was kindly provided by the Infectious Disease Research Institute (Seattle, WA, USA). For the $S$. japonicum infection model, there were two independent trials with two groups of mice (control and vaccinated) in each experiment. A total of 29 mice was utilized for the first vaccine trial divided into two groups of 14 mice (control) and 15 mice (vaccinated). The second trial followed similar pattern but with 10 mice in each group. Control mouse from each experiment was injected with a $100 \mu \mathrm{l}$ of $5 \mu \mathrm{g}$ GLA-SE while each mouse from the experimental groups was immunized with a $100 \mu$ of $25 \mu \mathrm{g} \mathrm{rSm}-\mathrm{p} 80$ formulated in $5 \mu \mathrm{g}$ GLA-SE. Each mouse was immunized three times at 4-week interval (weeks 0,4 , and 8) intramuscularly. For the $S$. haematobium infection model, a total of 30 hamsters was randomly divided into two groups (control and vaccinated) of 15 hamsters each. Each hamster in vaccinated group was injected intramuscularly with $400 \mu \mathrm{g}$ DNA vaccine (VR1020/Sm-p80) with $100 \mu \mathrm{g} \mathrm{CpG}$ oligonucleotides (ODN No. 101014) in right leg and $100 \mu \mathrm{g}$ protein vaccine (rSm-p80) with $100 \mu \mathrm{g} \mathrm{CpG} \mathrm{ODN} \mathrm{No.} 101014$ in the left leg while each hamster in control group received the same dose of delivery vector (VR1020) with control CpG ODN No. 2137. CpG ODN No. 101014 (TCG TCG TTT CGT CGT 
TTT GTC GTT) and CpG ODN No. 2137 (TGC TGC TTT TGT GCT TTT GTG CTT) were obtained from Invitrogen (Thermo Fisher Scientific, MA, USA).

\section{Experimental challenge and animal necropsy}

Schistosoma japonicum cercariae were collected by gently crushing infected $O$. hupensis snails to release the cercariae. Four weeks after the final immunization, each mouse from each group was challenged with 40 cercariae using the cover slip method (Tucker et al. 2013). All mice were euthanized 7 weeks post-cercarial challenge and adult worms recovered from the mouse hepatic portal system by perfusion were counted as already described (Tucker et al. 2013). For the hamster challenge, S. haematobium cercariae were collected from infected B. truncatus snails by shedding. Four weeks after the prime-boost vaccination, all hamsters were challenged with 200 cercariae via their shaved abdomen. Percent reduction in worm burden was determined by comparing the number of worms retrieved from the experimental group to the control group. In order to isolate trapped schistosome eggs, the livers, intestines, and bladder (hamster only) of the euthanized mice and hamsters were also collected and digested overnight in 5\% potassium hydroxide (Tucker et al. 2013). Egg burden in these tissues were determined as described previously (Ahmad et al. 2009a, b; Tucker et al. 2013; Zhang et al. 2010b).

\section{Antibody response following vaccination}

Blood samples were obtained from each mouse via cheek bleed and from each hamster via retro-orbital bleed prior to each immunization and prior to the cercarial challenge as well as at euthanasia. Serum samples were obtained by centrifugation after blood was allowed to clot at room temperature. Antibody response following vaccination was measured by enzyme-linked immunosorbent assay (ELISA) as previously described (Le et al. 2014; Zhang et al. 2010b). Briefly, 96-well plates were coated with $\mathrm{rSm}-\mathrm{p} 80$ (1.2 $\mu \mathrm{g} / \mathrm{well})$. Sm-p80specific antibody titers for total IgG subtypes, IgA, and IgM were determined using HRP-labeled anti-mouse secondary antibodies (Alpha Diagnostics Intl. Inc. TX, USA). Following the addition of the substrate, 3, 3',5,5'tetramethylbenzidine (TMB)-stabilized substrate (Promega, WI, USA), the samples were read at wavelength $450 \mathrm{~nm}$. The data obtained are reported as mean endpoint titers as previously described (Frey et al. 1998).

\section{Cytokine mRNA expression as determined by real-time PCR}

Following animal euthanasia, the spleen of each mouse and/or hamster was collected and splenocytes were isolated as previously described (Le et al. 2014). In brief, the spleens were gently crushed to release splenocytes and washed three times before resuspending cells in complete media (RPMI-1640 supplemented with $10 \%$ fetal bovine serum, $100 \mu \mathrm{g} / \mathrm{ml}$ penicillin $\mathrm{G}, 100 \mu \mathrm{g} / \mathrm{ml}$ streptomycin, and $10 \mu \mathrm{g} / \mathrm{ml}$ gentamycin). Cells were counted by trypan blue exclusion method and $1 \times 10^{6}$ cells/well were plated into 6-well plates. Seeded cells were incubated at $37{ }^{\circ} \mathrm{C}$ and $5 \% \mathrm{CO}_{2}$ for $24 \mathrm{~h}$ in the presence or absence of $12 \mu \mathrm{g}$ rSm-p80. Extraction of total RNA from cells was carried out using the RNeasy plus mini kit (Qiagen, CA, USA) according to manufacturer's instructions. First-strand cDNA synthesis was carried out using the cloned AMV reverse transcriptase (Thermo Fisher Scientific, MA, USA) according to manufacturer's instructions. Expression levels of a panel of Th1, Th2, and Th17 cytokines were determined by quantitative PCR (qPCR) using the StepOne ${ }^{\mathrm{TM}}$ plus Real-time PCR machine (Applied Biosciences, USA). GAPDH (glyceraldehyde 3phosphate dehydrogenase) was utilized as the housekeeping gene. The cytokine mRNA expression profile was determined by comparing the differences in the mRNA levels of the experimental group with the controls after normalization with GAPDH through the DataAssist ${ }^{\mathrm{TM}}$ software package V3.0 (Applied Biosciences, USA).

\section{Statistical analysis}

Differences between groups were assessed using the Student $t$ test. Statistical differences were defined as a $p$ value $<0.05$ using GraphPad Prism software (Version 7.03). All data are presented as the mean $\pm \mathrm{SE}$.

\section{Results}

Immunolocalization of Sm-p80/Sm-p80 ortholog proteins in Schistosoma mansoni, S. haematobium, and $S$. japonicum

The data from this current study showed that Sm-p80/Sm-p80 ortholog proteins are present at various locations in different development stages in all three species. Sm-p80/Sm-p80 ortholog proteins are distributed on the surface of eggs in all three species (Fig. 1 a1-c1). However, the distribution of Smp80/Sm-p80 ortholog proteins in cercariae is different among the species but mainly observed in the head (Fig. 2). In S. mansoni, Sm-p80 is localized to the acetabular gland in the head of the cercariae as well as in the duct (Fig. 2a1). In S. haematobium, the distribution of Sm-p80 ortholog protein is mainly observed in duct of the cercariae (Fig. 2b1). In S. japonicum, $\mathrm{Sm}-\mathrm{p} 80$ ortholog protein is located in the region of oral sucker, acetabular gland, and the duct (Fig. 2c1). These 


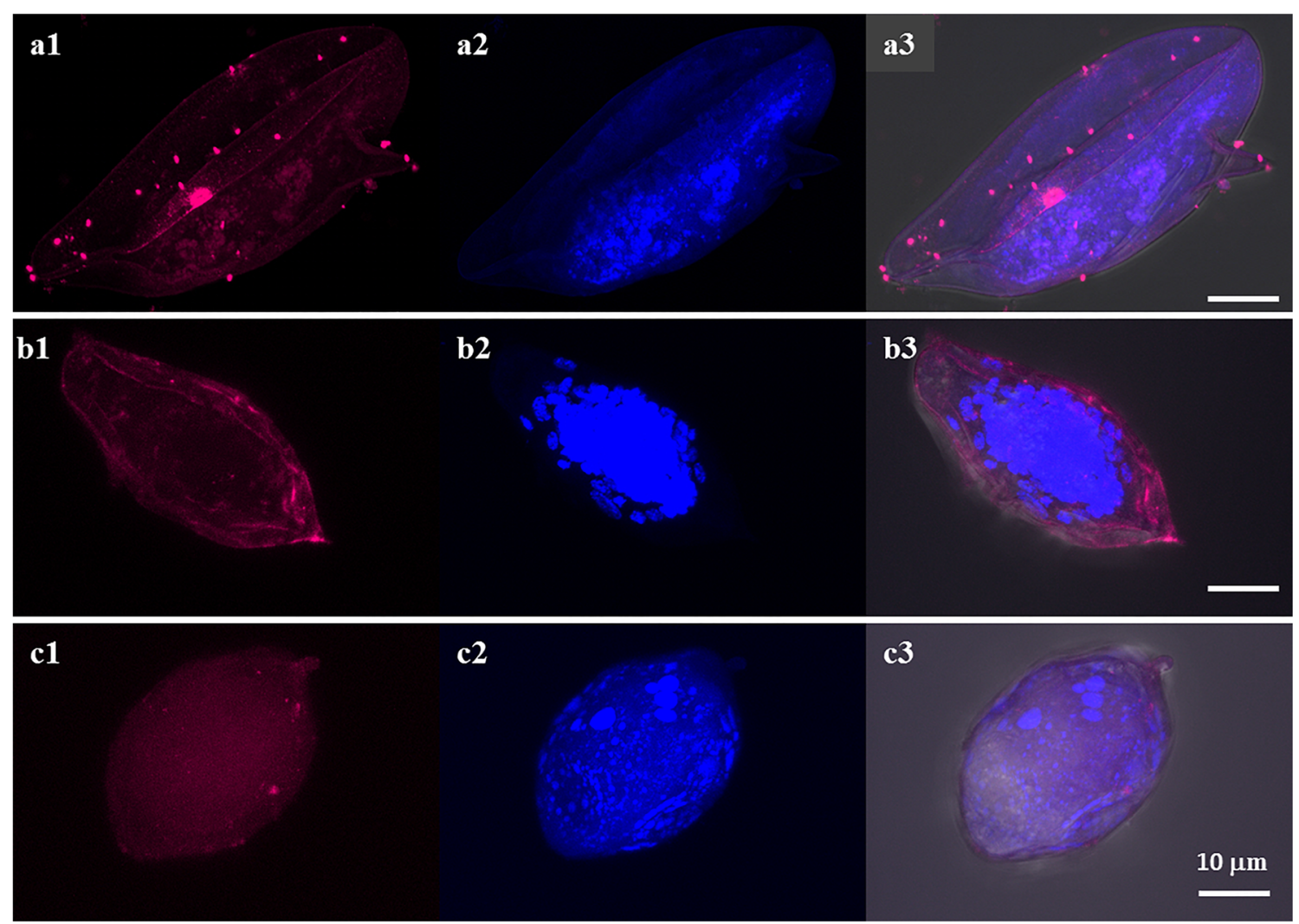

Fig. 1 Localization of Sm-p80 in Schistosoma mansoni, S. haematobium, and S. japonicum eggs. Representative fluorescence images of S. mansoni, $S$. haematobium, and $S$. japonicum eggs, respectively, are shown in panels $\mathrm{a}, \mathrm{b}$, and c. The distribution of Sm-p80/Sm-p80 orthologs is shown in a1, b1, and c1 images (magenta). The eggs were labeled with the rabbit antibody against Sm-p80. a2, b2, and c2 show DAPI labeling which stains nuclei (blue). The samples were mounted using Prolong Gold antifade reagent with DAPI. a3, b3, and c3 show an overlay showing the distribution of sm-p80/Sm-p80 orthologs (magenta) and DAPI (blue) in differential interference contrast (DIC). The images were taken using a Nikon T1E confocal microscopy with a $60 \times$ objective and analyzed with NIS software. The eggs images represent a maximum projection intensity derived from a Z-stack. Scale bars, $10 \mu \mathrm{m}$

\section{Sm-p80 vaccine formulations induced cross-species protection}

In order to determine the cross-species prophylactic potential of the Sm-p80 formulated in GLA-SE against S. japonicum infections in mice, an immunization regimen was tested in two independent trials as described above. The Sm-p80-based vaccine showed a significant prophylactic effect resulting in the reduction of worms in vaccinated group when compared to the control animals. Specifically, we observed an aggregate mean of $46.75 \%$ reduction in worm burden (Trial $1-28.69 \%$, Trial $2-55.11 \%$ ) in mice following vaccination with $\mathrm{rSm}-\mathrm{p} 80 /$ GLA-SE $(p=0.02)$ (Fig. 5a and Table 1). There was a $26.9 \%$ reduction in worm numbers in hamsters vaccinated with the Sm-p80 DNA vaccine and boosted with recombinant protein vaccine ( $p=0.14$ ) (Fig. 6a). Egg load in the liver and intestines was determined for both mouse groups (experimental and control). The control group (mean egg count $=9947.88 \pm 565.44 \mathrm{eggs} / \mathrm{g}$ of liver) compared with the Sm-p80/GLA-SE formulation group (mean egg count $=9475.93 \pm 667.63 \mathrm{eggs} / \mathrm{g}$ of liver $)$ in both with each experiment. 

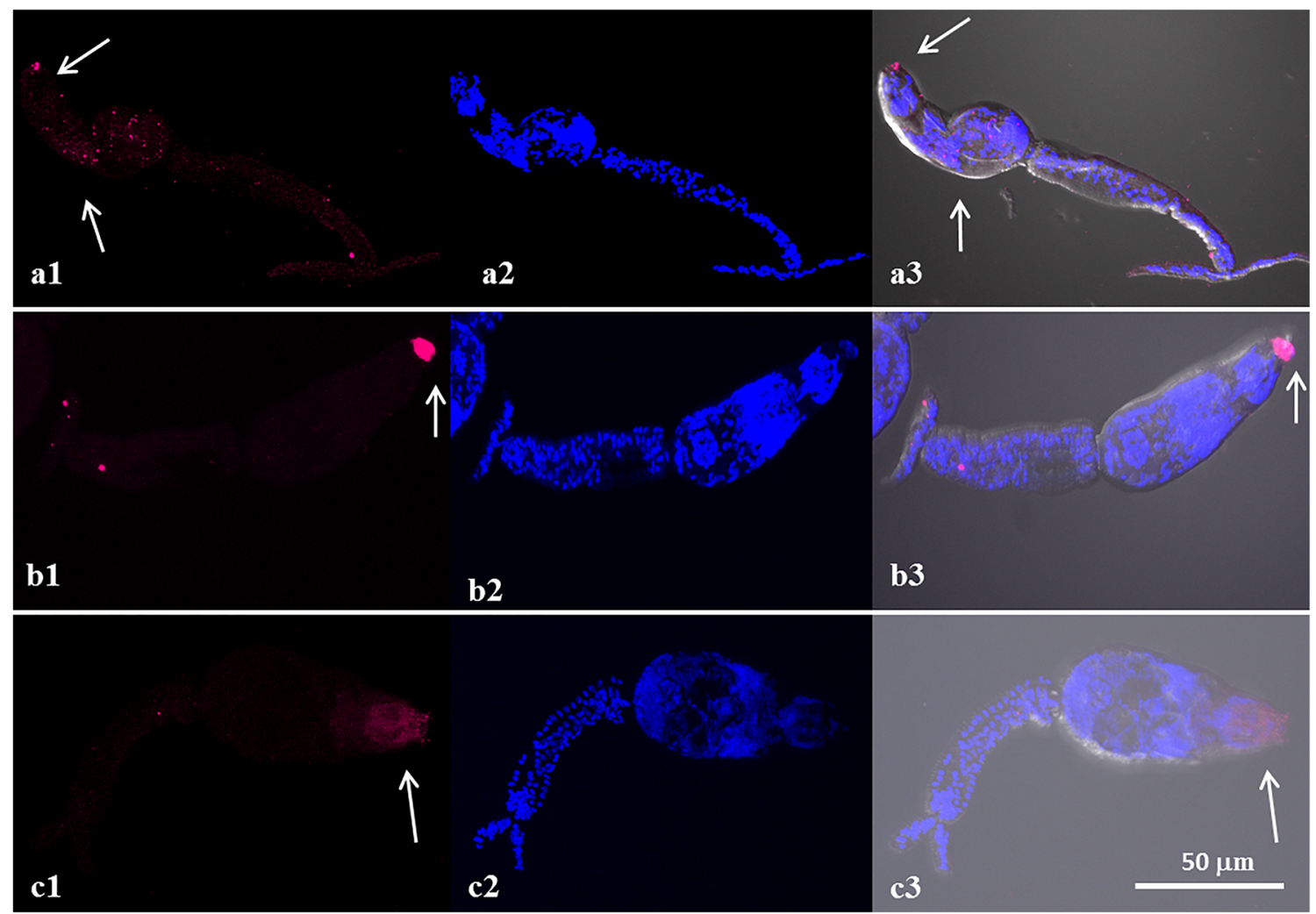

Fig. $2 \mathrm{Sm}-\mathrm{p} 80 / \mathrm{Sm}-\mathrm{p} 80$ ortholog is distributed in the head of Schistosoma mansoni, S. haematobium, and S. japonicum cercariae. Representative fluorescence images and merged with differential interference contrast (DIC) of S. mansoni, S. haematobium, and $S$. japonicum cercariae, respectively, are shown in panels a, b, and c. Sm-p80 and Sm-p80 ortholog protein localization is shown in a1, b1, and c1which is mainly observed in the head in the three species (arrows). In $S$. mansoni, Sm-p80 is located mostly in the head of the cercariae as well as in the duct (a1). In S. haematobium, Sm-p80 ortholog protein is mainly observed in duct (b1). In S. japonicum, Sm-p80 ortholog protein is

S. japonicum challenge trials revealed a mean $4.74 \%$ reduction (Trial $1-0.98 \%$, Trial $2-10.2 \%$ ) in hepatic egg burden $(p=0.86)$ (Fig. 5b and Table 1). However, no reduction in intestinal egg burden using the cumulative data from two trials showed a difference in egg load between the control (mean egg count $=44,621.98 \pm 3286.9$ eggs $/ g$ of intestine) and the vaccinated group (mean egg count $=46,565.94 \pm 2388.33$ eggs/g of intestine) (Fig. 5c and Table 1). However, Trial 2 showed a reduction of $39.9 \%$ in eggs trapped in intestines whereas Trial 1 had showed no difference. No reduction in liver and intestine egg load was observed in hamsters vaccinated with the prime-boost vaccine (Fig. $6 \mathrm{~b}$, c, Table 2). No eggs were detected in the urinary bladder of hamsters from either the control or experimental groups.

\section{Anti-Sm-p80 specific antibody response following vaccination}

Antibody production patterns were compared between the control and experimental mouse groups using the sera located in the region of oral sucker, head gland, and the duct (c1). These observations suggested that calpain is secreted from cercariae. The cercariae were labeled with the rabbit antibody against Sm-p80 (magenta). The samples were mounted using Prolong Gold antifade reagent with DAPI. a2, b2, and c2 show DAPI which stains nuclei (blue). a3, b3, and c3 show an overlay of the distribution of sm-p80 (magenta) and DAPI (blue) in DIC. The images were taken using a Nikon T1-E confocal microscopy with a $40 \times$ objective and analyzed with NIS software. The cercariae images represent a maximum projection intensity derived from a Z-stack. Scale bars, $50 \mu \mathrm{m}$

collected throughout the immunization regimen. Anti-Smp80-specific antibodies (total $\operatorname{IgG}$ and $\operatorname{IgG}$ subtypes) were not detected in control mice sera. In both $S$. japonicum infection model trials, there was an increase in Sm-p80specific total IgG titers following second immunization at week 4 in vaccinated mice with peak endpoint titers of 409,600 at week 6 (Fig. 7a) which remained high until experimental challenge (week 12). Robust antibody responses were also observed for IgG1 (end point titer 102,400), IgG2a and IgG2b (end point titer 12,800), IgG3 (end point titer 6400) as well as for IgA (end point titer 6400 ), and IgM (end point titer 12,800) in mice vaccinated with Sm-p80/GLA-SE (Fig. 7a). Interestingly, we observed a marked increase in the total IgG antibody titer presacrifice suggesting that calpain antigens released (among many other antigens) during parasite killing might have served as a booster for the memory B cells generated through vaccination. Robust antibody responses were also observed for total $\operatorname{IgG}$ (end point titer, 6400) in vaccinated hamsters which remained high until euthanasia (Fig. 7b). 


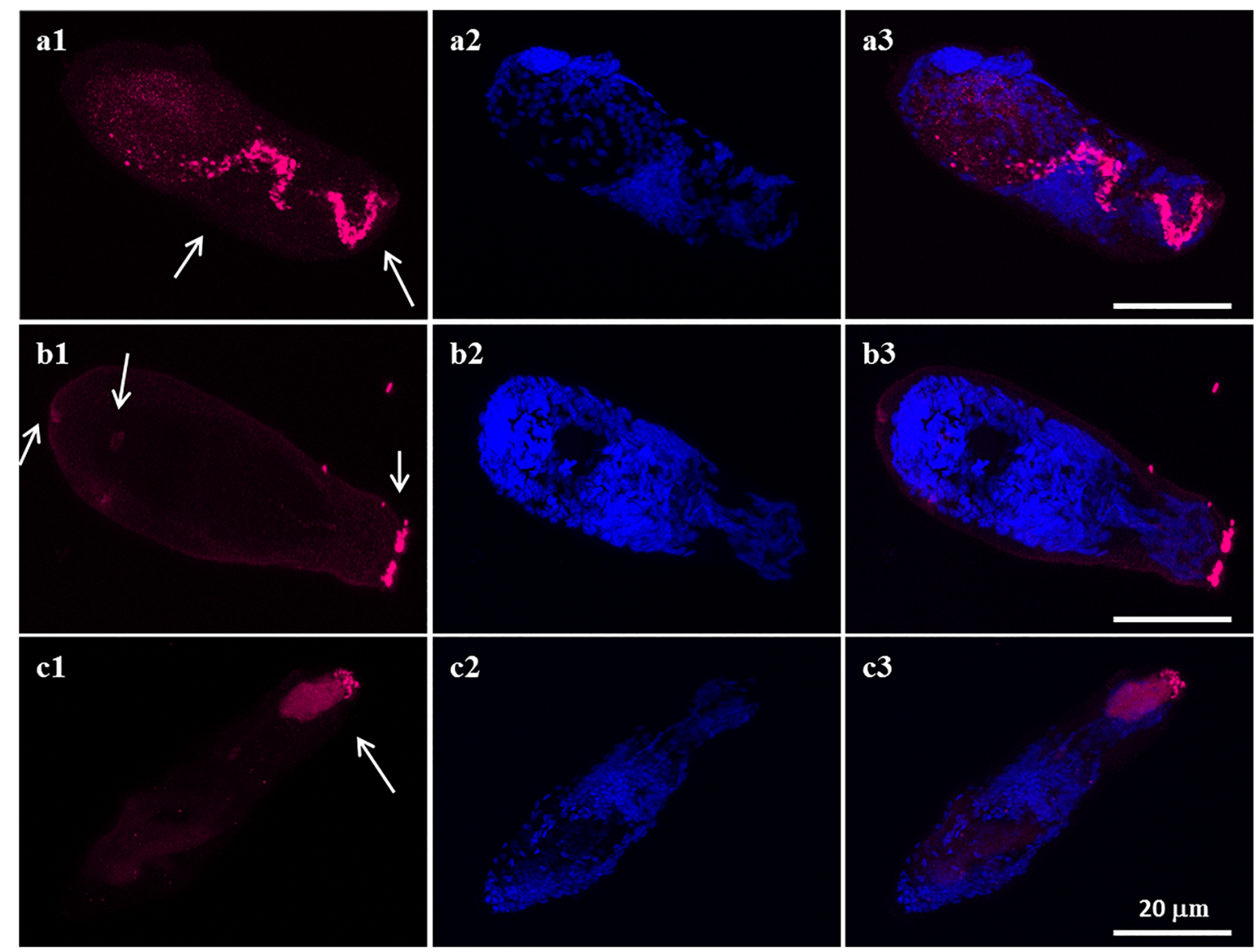

Fig. $3 \mathrm{Sm}-\mathrm{p} 80 / \mathrm{Sm}-\mathrm{p} 80$ ortholog protein is present on the tegument in the three species larvae. Representative fluorescence images of Schistosoma mansoni, S. haematobium and S. japonicum schistosomula, respectively, are shown in panels a, b, and c. a1, b1, and c1 show the distribution of smp80 and Sm-p80 ortholog proteins in the tegument. In addition, it is localized in vesicle within the schistosomulae of $S$. mansoni (a1, arrows). However in S. haematobium, Sm-p80 is mainly located in the duct as well as in the acetabulum and the excretory pore (b1, arrows). In S. japonicum, the expression pattern of Sm-p80 is in the region of the oral sucker and the

\section{Cytokine production in mice and hamster splenocytes following Sm-p80 stimulation}

Following $24 \mathrm{~h}$ incubation of splenocytes isolated from control and vaccinated animals with concurrent stimulation with rSm-p80, the message expression of a panel of cytokines was analyzed by qRT-PCR. The cytokine mRNA profiles are summarized in Fig. 7. In both $S$. japonicum infection trials, there was a significant increase in Th1 cytokines production in mice immunized with Sm-p80/GLA-SE when compared to the controls. The observed increase in Th1 cytokine production was exemplified by the observed significant increase in the production of IFN- $\gamma$ in mice immunized with Sm-p80 when compared to the adjuvant control group $(p<0.05)$. There were also significant increases in other Th1 cytokines levels such as $\mathrm{TNF} \alpha$, IL-2, and IL-12 in experimental group compared to the control group (Fig. 7c). Th2 cytokine levels were also analyzed. We observed a statistical significant increase in IL- duct (c1, arrows). The schistosomula were labeled with the rabbit antibody against sm-p80 and Sm-p80 ortholog proteins (magenta). a2, b2, and c2 show DAPI labeling (blue). a3, b3, and c3 show an overlay of the distribution of Sm-p80/Sm-p80 ortholog protein (magenta) and DAPI (blue). The images were taken using a Nikon T1-E confocal microscopy with a $60 \times$ objective and analyzed with NIS software. The schistosomula images represent a maximum projection intensity derived from a Z-stack. Scale bars, $20 \mu \mathrm{m}$

4 levels in the experimental animals when compared to their control counterparts. However, IL-5 production appeared downregulated in both experimental and control groups but no significant difference was observed between these groups (Fig. 7c). The levels of IL-17 cytokine were also determined to evaluate the possible role(s) for Th17-type immune responses in protective immunity. Our result showed that IL-17 was downregulated in both control and experimental groups but not significant. A similar trend of upregulation of Th1- and Th2-dependent cytokines was observed in splenocytes from vaccinated hamsters stimulated in vitro with $\mathrm{rSm}-\mathrm{p} 80$ antigen (Fig. 7d).

\section{Discussion}

The immunolocalization analysis showed that Sm-p80/Smp80 ortholog proteins are distributed at various locations in 

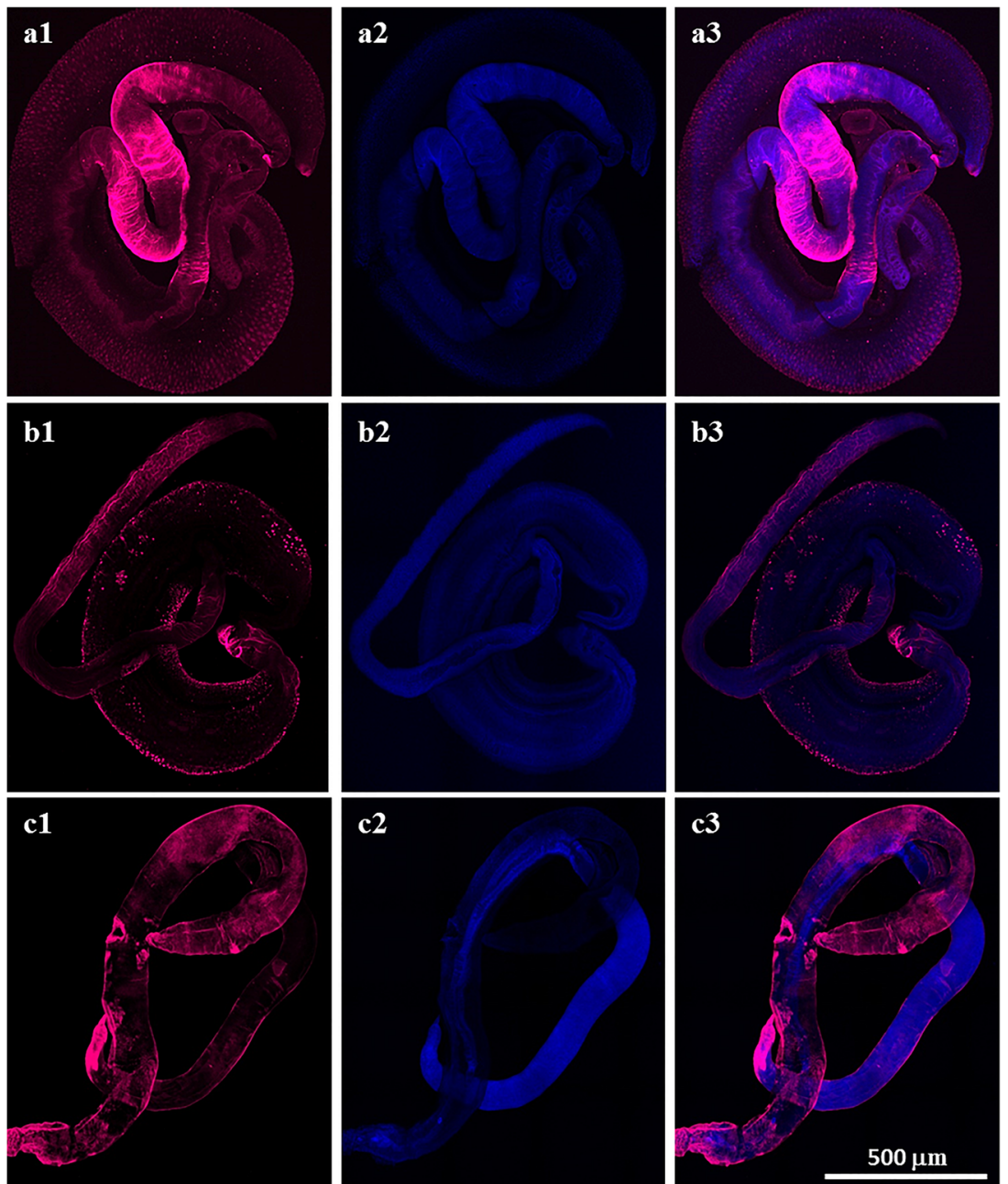

c2

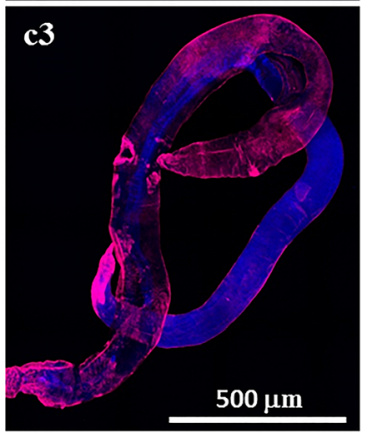

Fig. $4 \mathrm{Sm}-\mathrm{p} 80 / \mathrm{Sm}-\mathrm{p} 80$ ortholog protein is localized on the tegument of Schistosoma mansoni, S. haematobium, and S. japonicum adult worms. Representative stitched fluorescence images of $S$. mansoni, $S$. haematobium, and $S$. japonicum adult worms, respectively, are shown in panels a, b, and c. al, b1, and c1 show the distribution of smp80 and Sm-p80 ortholog proteins. The $S$. mansoni adult worms were labeled with the rabbit antibody against $\mathrm{Sm}-\mathrm{p} 80$ (a1), the $S$. haematobium with the mice serum against Sm-p80 ortholog protein (b1) and the

S. japonicum with the baboon serum against Sm-p80 ortholog protein (c1). The samples were mounted using Prolong Gold antifade reagent with DAPI. a2, b2, and c2 show DAPI labeling which stains nuclei (blue). $\mathrm{a} 3, \mathrm{~b} 3$, and $\mathrm{c} 3$ show an overlay of the distribution of sm-p $80 / \mathrm{Sm}-\mathrm{p} 80$ ortholog proteins (magenta) and DAPI (blue). The images taken using a Nikon T1-E confocal microscopy with a $10 \times$ objective were stitched and analyzed with NIS software. All images represent a maximum projection intensity derived from a Z-stack. Scale bars, $500 \mu \mathrm{m}$

a

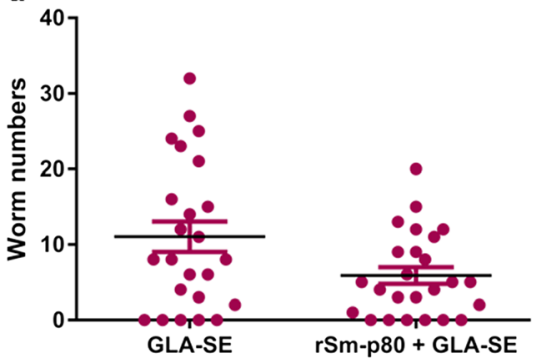

b

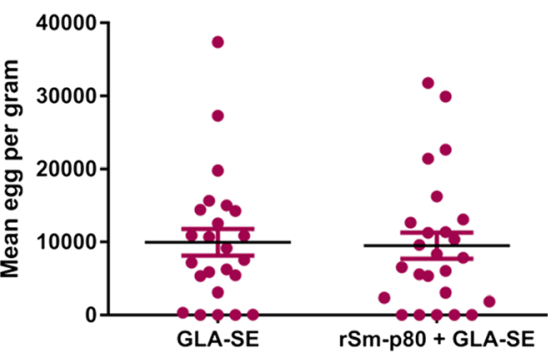

c

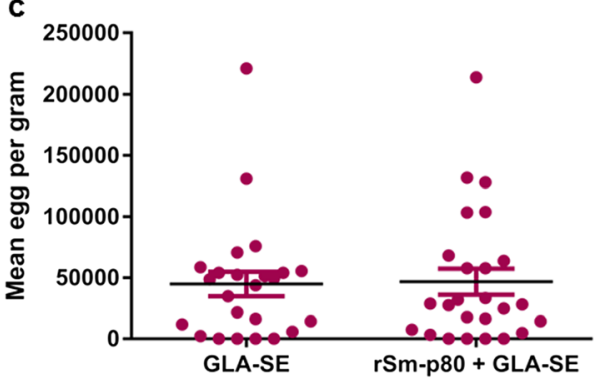

Fig. 5 Worm and tissue egg burden. a Worm numbers per mouse are represented for all mice (Trials 1 and 2 combined) in the GLA-SE control group and the experimental group (rSm-p80 + GLA-SE) $(p=0.02)$. Cumulative egg load per gram of liver for both trials $(p=0.86)(\mathbf{b})$ and

egg load per gram of intestine ( $p=0.89)$ (c). Worm burden and tissue egg load determined 7 weeks following Schistosoma japonicum cercarial challenge. Each mouse was challenged with $40 \mathrm{~S}$. japonicum cercariae. Double asterisks represent statistical significance, $\mathrm{ns}=$ not significant 
Table 1 Reduction in Schistosoma japonicum worm numbers and eggs in tissue of mice following vaccination with Sm-p80 vaccine

\begin{tabular}{|c|c|c|c|c|c|c|}
\hline \multirow[t]{2}{*}{ Group } & \multirow[t]{2}{*}{ Worm burden $($ mean \pm SE) } & \multicolumn{2}{|c|}{ Tissue egg burden per gram } & \multirow[t]{2}{*}{ Worm reduction } & \multicolumn{2}{|c|}{ Tissue egg reduction } \\
\hline & & Liver & Intestine & & Liver & Intestine \\
\hline $\begin{array}{l}\text { GLA-SE }(n=24) \\
\text { rSm-p } 80+\text { GLA-SE }(n=25)\end{array}$ & $\begin{aligned} 11.04 & \pm 2.01 \\
5.88 & \pm 1.1\end{aligned}$ & $\begin{array}{l}9947.88 \pm 565.44 \\
9475.93 \pm 667.63\end{array}$ & $\begin{array}{l}44,621.98 \pm 3286.90 \\
46,565.94 \pm 2388.33\end{array}$ & $46.75 \%$ & $4.74 \%$ & No protection \\
\hline
\end{tabular}

different developmental stages of all three schistosome species as well as on the tegument of the adult worms indicating that native Sm-p80 from S. mansoni, and Sm-p80 ortholog proteins from $S$. japonicum and $S$. haematobium can be recognized by anti-Sm-p80-specific antibodies. The role(s) of Sm-p80/Sm-p80 ortholog proteins in each of the developmental stages of schistosomes is not yet completely understood. Immunohistochemistry of $S$. mansoni adult worms showed that $\mathrm{Sm}-\mathrm{p} 80$ is localized to the surface syncytial epithelium and the underlying musculature which is in agreement with our previous study (Siddiqui et al. 1993). Our study also showed that $\mathrm{Sm}-\mathrm{p} 80$ ortholog protein was expressed on the tegument of both adult male and female worms of $S$. japonicum which is in contrast to a previous study by Kumagai et al. (2005) who reported that calpain was only expressed on the tegument of $S$. japonicum adult male worm. Furthermore, we observed that $\mathrm{Sm}$-p80 ortholog protein is also localized to the surface of S. haematobium adult worms. To our knowledge, this is the first study to demonstrate the expression profile of $\mathrm{Sm}-\mathrm{p} 80$ ortholog proteins in the developmental stages of $S$. haematobium. The localization of Smp80 ortholog protein to the secretion glands of the cercariae suggests a possible role for this protein(s) in the penetration of host skin during infection. Studies by others (Jankovic et al. 1996; Kumagai et al. 2005; Ohta et al. 2004) have demonstrated the presence of calpain in secreted contents of cercariae and newly transformed schistosomulae.

Due to its critical role in schistosome membrane renewal and/or biogenesis as well as its role in host immune evasion, our group has focused on systematic development of Sm-p80 as a schistosomiasis vaccine candidate. Over the last two decade, we have employed a systematic approach to developing Sm-p80-based vaccine for schistosomiasis control. Several studies by our group using various Sm-p80-based vaccination strategies have demonstrated high levels of prophylactic and therapeutic efficacy against $S$. mansoni infections in both mice and baboons (Ahmad et al. 2009a, b, c; Karmakar et al. 2014a, b; Le et al. 2014; Zhang et al. 2010b, 2011); efficacy levels obtained with Sm-p80 vaccine are comparable to previously obtained data with $S$. mansoni radiationattenuated (RA) cercariae vaccine, which is a gold standard for anti-schistosome protective immunity. Immunizations with the RA vaccine have been demonstrated to show significant protection levels ranging from $56 \%$ to as high as $80 \%$ following cercariae challenge (Anderson et al. 1999; GanleyLeal et al. 2005; Sher et al. 1982; Wilson et al. 1999). Based on the findings from our recent study which evaluated the cross-species protective efficacy of Sm-p80-based vaccine against urinary schistosomiasis in hamsters and baboons in which Sm-p80 vaccine offered significant protection against S. haematobium infections (Karmakar et al. 2014a), we hypothesized that protection against Asiatic schistosomiasis caused by $S$. japonicum infections would be possible by inducing specific host immune responses through Sm-p80based vaccination. The data presented here from the two independent $S$. japonicum vaccine trials showed that $\mathrm{Sm}-\mathrm{p} 80$ vaccine conferred significant cross-species protection against Asiatic schistosomiasis in mice. In this study, we have demonstrated that mice vaccinated with Sm-p80 formulated in GLA-SE had a significant reduction in worm numbers when compared to animals that received adjuvant only. A possible explanation for the variability in adult worm recovery post- a

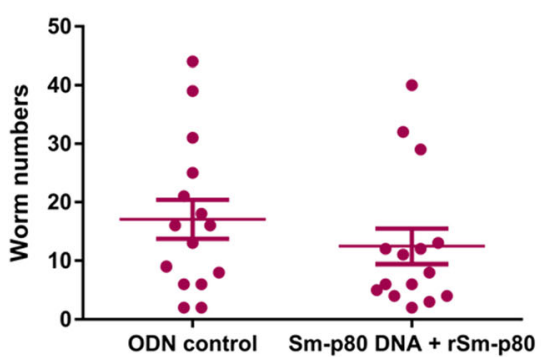

Fig. 6 Worm and tissue egg burden. a Worm numbers per hamster. $(p=0.14) \mathbf{b}$ Egg burden per gram of liver, $(p=0.7503)$ and $\mathbf{c}$ egg burden per gram of intestine $(p=0.9846)$ are shown for hamster in the CpG ODN control group and experimental group (Sm-p80 DNA + rSm- b

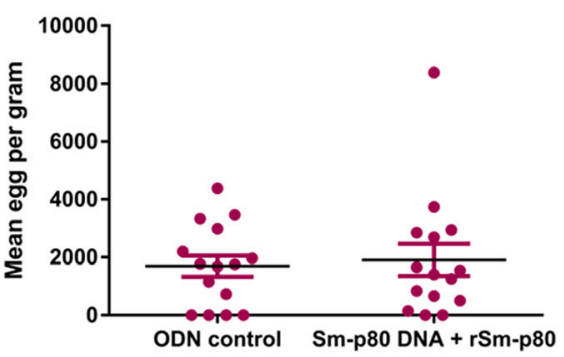

C

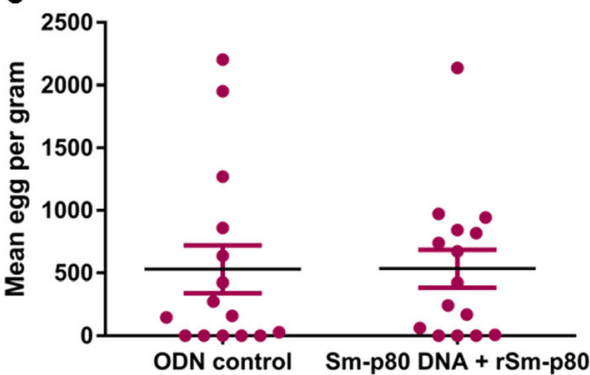

p80). Each hamster was challenged with 200 S. haematobium cercariae. Worm burden and tissue egg load determined 12 weeks following Schistosoma haematobium cercarial challenge. ns represents not significant 
Table 2 Reduction in

Schistosoma haematobium worm numbers in hamsters following vaccination with $\mathrm{Sm}-\mathrm{p} 80$ vaccine

\begin{tabular}{lllll}
\hline Group & $\begin{array}{l}\text { Worm burden } \\
(\text { mean } \pm \text { SE) }\end{array}$ & \multicolumn{2}{l}{ Tissue egg burden per gram } & Worm \\
\cline { 3 - 4 } & & Liver & Intestine & \\
\hline CpG ODN $(n=15)$ & $17.07 \pm 3.34$ & $1435.26 \pm 300.57$ & $530.27 \pm 148.23$ & $26.95 \%$ \\
$\begin{array}{l}\text { Sm-p80 DNA + rSm-p80 } \\
(n=15)\end{array}$ & $12.47 \pm 3.02$ & $1647.42 \pm 512.95$ & $534.98 \pm 86.81$ & \\
\hline
\end{tabular}

euthanasia between the two trials may not be unconnected with the challenges associated with experimental infection of animals with $S$. japonicum cercariae as these parasites are not easily manipulated like their S. mansoni and S. haematobium counterparts due to their extreme sticky nature (Tucker et al. 2013; Wang et al. 2006). The protective efficacy reported in this present study with Sm-p80 formulated in GLA-SE is beyond that obtained with radiation-attenuated $S$. japonicum cercariae vaccine in another study (Osada et al. 2001; Zhang et al. 2010a) and even more noteworthy is the fact that the Sm-p80 vaccine antigen utilized in this study was identified from another species of schistosome, S. mansoni. Studies utilizing prime-boost vaccination strategies using several schistosome antigens (Ahmad et al. 2009c; Le et al. 2014) have shown encouraging outcomes as well as in other infectious disease models (Dale et al. 2006; De Rose et al. 2006; Schneider et al. 2001; Tanghe et al. 2001a, b). In the S. haematobium/hamster model, in which we explored the protective efficacy of a prime-boost vaccination strategy (same-day administration of both DNA and protein vaccine), our results showed that hamsters vaccinated with the prime-boost vaccine had a considerable reduction in adult worm numbers but not as significant as we have previously reported with a different Sm-p80based vaccination strategy in which the vaccinated animals received three immunizations (primary plus two boosters) prior to challenge with cercariae (Karmakar et al. 2014a). The lower worm burden observed in this current study suggests that a same-day vaccine administration without boosters might not be the ideal strategy against $S$. haematobium infections despite the promising result we had previously obtained with $S$. mansoni/mouse infection model using the same primeboost strategy (Ahmad et al. 2009c).

Reduction in egg burden is of a particular importance as the immunopathogenesis of schistosomiasis is associated with the host immune responses to antigens secreted by eggs trapped within host tissues (Colley et al. 2014; Colley and Secor
Fig. 7 Humoral and Cellular immune responses following vaccination. Titers of anti-Smp80 specific antibodies in vaccinated mice (a) and hamsters (b). The peak antibody titers for IgG, IgG subtypes, $\operatorname{IgA}$, and $\operatorname{IgM}$ are shown for immunized mice and total IgG for immunized hamsters. Th1, Th2, and Th17 cytokine mRNA transcripts produced $24 \mathrm{~h}$ post stimulation of isolated mice splenocytes with rSm-p80 (c). Th1 and Th2 cytokine production in stimulated hamster splenocytes (d). Stimulated splenocytes were pooled splenocytes isolated from each mouse or hamster belonging to each group (control and vaccinated). Cytokine relative expression was analyzed by real-time PCR
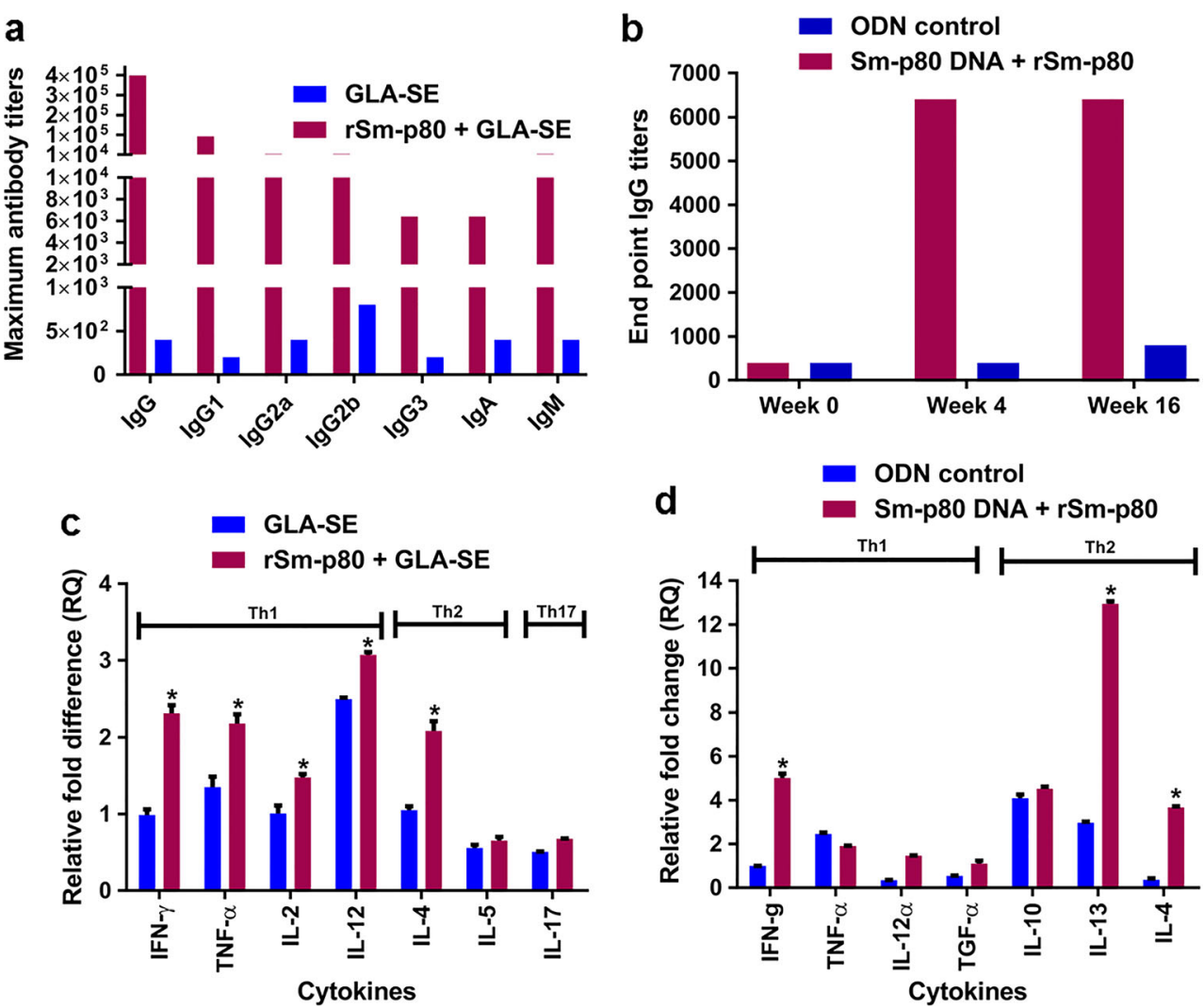
2014). Reduction in intestinal egg burden within the host may be associated with a decrease in disease transmission because lower amount of schistosome eggs are released by the host into the environment (Ricciardi et al. 2016). Therefore, an effective schistosomiasis vaccine should not only target reduction in worm burden but also the reduction in eggs trapped in host tissue and/or excreted. In Trial 1 (S. japonicum infection model), there was no reduction in either the hepatic or the intestinal egg burden in mice immunized with Sm-p80 when compared to the control which might be due to the fact that there was no difference in number of female worms recovered between both groups after perfusion. This argument is further strengthened by the results obtained in the Trial 2 in which we observed moderate reduction in hepatic egg load in immunized mice (reduction of $10 \%$ compared to controls) and a much higher reduction in intestinal egg burden (reduction of $39 \%$ compared to controls). This observed reduction in tissue egg load in animals vaccinated with Sm-p80 is associated with the vaccine-mediated killing of the adult female worms which was $22 \%$ higher in Trial 2. In addition, a decrease in tissue egg burden would ultimately lead to a decrease in disease transmission as fewer eggs are passed into the environment by the infected host. Surprisingly, there was no reduction in liver and intestine tissue egg load in hamsters vaccinated with Sm-p80. We expect to further investigate this in our future planned studies. Contrary to other reports (Ghandour 1978; Vuong et al. 1996), no eggs were observed in the urinary bladders of the hamsters in the control group or the vaccinated group. This drawback further supports the argument that hamster is not an ideal animal model to fully study urogenital schistosomiasis (Honeycutt et al. 2014; Rinaldi et al. 2015). The data presented here on cross-species efficacy of Sm-p80 against $S$. japonicum and $S$. haematobium infections in addition to our previously published vaccine efficacy studies against $S$. mansoni infections strengthens the paradigm that Sm-p80based vaccine could be useful in the control of the three major species of schistosomes that infect humans thereby reducing the overall global burden of the disease.

The importance of specific IgG antibodies in protection against schistosomiasis has been demonstrated by our studies and others. Sera obtained from donor-immunized mice and baboons have been shown to protect recipients against subsequent schistosome challenge when passively transferred (Byram et al. 1979; Doenhoff et al. 1979; Mangold and Dean 1986; Melo et al. 2014; Torben et al. 2011). Studies utilizing schistosome non- and/or semi-permissive host such as rats have shown that resistance to schistosome infections as well as mechanism of schistosome killing are almost entirely humoral mediated by high IgG antibodies (Knopf et al. 1977; Wilson et al. 2008). Our group has also demonstrated the potential effector role of antibody-dependent complement mediated cytotoxicity (ADCC) in schistosome killing (Torben et al. 2012). In both $S$. japonicum challenge trials, immunizations with Sm-p80 formulated in GLA-SE elicited significant production of Sm-p80-specific antibody in immunized mice demonstrating that the vaccine formulation had a substantial impact on humoral immunity which appeared to correlate with the protection observed. We also observed significant differences in endpoint antibody titers when comparing IgG subclasses within the experimental group of mice. IgG1 production was significantly higher than the other IgG subtypes. This is not surprising as IgG1 production is associated with T-helper cell 2 (Th2) phenotype in mice being stimulated by IL-4 while IgG2a and IgG2c are predictors of Th1 type of immune response in these animals (Martin et al. 1998; Mosmann and Coffman 1989). IgG1 production has been associated with protection against schistosomiasis (Karmakar et al. 2014a). Similarly, appreciable antibody titers of $\operatorname{IgG}$ were observed in prime-boost vaccinated hamsters when compared to the controls which appeared to correlate with the protection observed.

Th1 type immune responses are known to play major roles in protection against schistosomiasis. For instance, the resistance against schistosomiasis in individuals that are naturally resistant to schistosome infection (Endemic normal) is associated with Th1 immune response as demonstrated by high level production of interferon gamma (IFN- $\gamma$ ) by peripheral blood mononuclear cells (PBMCs) from these individuals stimulated with schistosome antigens (McManus and Loukas 2008; Viana et al. 1994). Furthermore, studies with RA vaccine showed that schistosomulae are killed in the lungs of protected animals as a result of inflammation (Wilson et al. 2016). In the RA model, co-administration of IL-12 (a potent Th1 inducing cytokine) with RA vaccine resulted in almost sterile immunity which was associated with a high induction of IFN- $\gamma$ in an autocrine manner (Wilson and Coulson 2009; Wilson et al. 1999, 2016). Studies by our group and other colleagues have shown that the production of IFN- $\gamma$ is associated with protection against schistosome infection in animal models (Ahmad et al. 2009c; Le et al. 2014; Pearson et al. 2012; Varaldo et al. 2004; Zhang et al. 2011). The protective role of TNF- $\alpha$ against schistosomiasis has also been demonstrated by the RA vaccine model (Street et al. 1999). IFN- $\gamma$ and TNF- $\alpha$ are also known to play a role in the activation of macrophages. In this current study, Sm-p80 formulated in GLA-SE significantly increased the production of Th1 cytokines, IFN- $\gamma$, TNF- $\alpha$, IL-2, and IL-12 (discussed above), in vaccinated animals when compared to the controls. We also observed an increased production of Th2-type cytokines such as IL-4 in stimulated splenocytes from vaccinated mice. Studies have shown that a balanced Th1/Th2 immune responses are required for protection against schistosomiasis and that Th2 responses are needed to achieve this protection (Pearce and MacDonald 2002). Induction of Th2 responses are thought to be associated with the recruitment of basophils and eosinophils which are thought to be involved in schistosomulae 
killing (El Ridi and Tallima 2013). Our results showed that Sm-p80 vaccine formulated in GLA-SE induced mixed Th1 (IFN- $\gamma$, TNF- $\alpha$, IL-2, and IL-12) and Th2-type immune responses which appeared to correlate with the cross-species protection observed in this $S$. japonicum model of infections which was in agreement with our previous studies on S. mansoni infection models (Karmakar et al. 2014a, b). The downregulation of IL-17 observed in the S. japonicum/mouse studies (Trials 1 and 2) might not be entirely non-beneficial as studies have shown that blocking IL-17 cytokine production in S. japonicum-infected mice resulted in the reduction of granuloma formation and fibrosis (Chen et al. 2013; Zhang et al. 2012). Th1 and Th2 cytokines regulation in the vaccinated hamsters followed similar pattern as those in the $S$. japonicum infection models as we observed upregulation of Th1 cytokines (IFN- $\gamma$, TNF- $\alpha$, and IL-12) and Th2 cytokines (IL-4, IL-10, and IL-13) in vaccinated animals (Fig. 7c, d).

The data presented in this current study are promising, thereby promoting the continued development of Sm-p80 as a single schistosomiasis vaccine with a potential of protecting against the three main species of schistosomes that infect humans. In the future, the Sm-p80 vaccine formulation can potentially be tested in a water buffalo model of Asiatic schistosomiasis as our group has previously done with nonhuman primate S. mansoni and S. haematobium infection models of the disease. Water buffaloes are responsible for over $75 \%$ of schistosomiasis transmission in China and Indonesia (Angeles et al. 2015; McManus et al. 2010) while carabao is largely responsible for the transmission in The Philippines (Gordon et al. 2012). Nonhuman primates, such as baboons, are natural host for schistosomiasis with similar clinical presentations to that in humans, and the data from such studies are more clinically applicable than data from rodent models. The data presented on same-day heterologous prime-boost vaccination indicate a robust induction of vaccine-induced specific immune responses which are comparable to standard strategies of multiple round of immunization. This approach saves time and could prove advantageous with regards to compliance.

In conclusion, the data presented in this study demonstrated that $\mathrm{Sm}-\mathrm{p} 80$ vaccine conferred cross-species protection against $S$. japonicum and $S$. haematobium infections in rodents. Sm-p80 vaccine elicited robust host immune responses which resulted in a balanced Th1/Th2 response associated with protection. The balanced $\mathrm{Th} 1 / \mathrm{Th} 2$ responses essential for both parasite killing and host-beneficial injury repair were elicited. The present study along with our previous published reports on the prophylactic and therapeutic efficacies of Smp80 vaccine against $S$. mansoni and $S$. haematobium infections supports Sm-p80 as a leading schistosomiasis vaccine with potential to protect against both hepatic/intestinal and urogenital schistosomiasis. However, further efficacy studies in large animal models, baboons and water buffaloes/cattle, are required to improve on the cross-species protective efficacy of the Sm-p80 vaccine against urogenital schistosomiasis and Asiatic schistosomiasis, respectively.

Acknowledgements We would like to thank Dr. Don McManus for providing us with adult worms and eggs of Schistosoma japonicum and Dr. Govert van Dam and Dr. C.H. Hokke for providing us with S. haematobium life cycle stages. We also thank Dr. Steve Reed and Dr. Darrick Carter of IDRI for providing GLA-SE adjuvant for these studies. The snails were supplied through a NIH-NIAID contract (HHSN2722010000051) to Biomedical Research Institute.

Funding This work was supported in part by grants from the Bill and Melinda Gates Foundation grant (OPP1097535) and from the National Institute of Allergy and Infectious Diseases (NIAID/NIH) SBIR (R43/ R44 AI103983).

\section{Compliance with ethical standards}

Conflicts of interests The authors declare that they have no conflict of interest.

Ethical approval All applicable international, national, and/or institutional guidelines for the care and use of animals were followed. All animal procedures were conducted in accordance with Institutional Animal Care and Use Committee (IACUC) Guidelines and were approved by the Animal Ethics Committee at Texas Tech University Health Sciences Center.

\section{References}

Ahmad G, Torben W, Zhang W, Wyatt M, Siddiqui AA (2009a) Sm-p80based DNA vaccine formulation induces potent protective immunity against Schistosoma mansoni. Parasite Immunol 31(3):156-161. https://doi.org/10.1111/j.1365-3024.2008.01091.x

Ahmad G et al (2009b) Protective and antifecundity effects of Sm-p80based DNA vaccine formulation against Schistosoma mansoni in a nonhuman primate model. Vaccine 27(21):2830-2837. https://doi. org/10.1016/j.vaccine.2009.02.096

Ahmad G et al (2009c) Prime-boost and recombinant protein vaccination strategies using Sm-p80 protects against Schistosoma mansoni infection in the mouse model to levels previously attainable only by the irradiated cercarial vaccine. Parasitol Res 105(6):1767-1777. https://doi.org/10.1007/s00436-009-1646-Z

Anderson S, Coulson PS, Ljubojevic S, Mountford AP, Wilson RA (1999) The radiation-attenuated schistosome vaccine induces high levels of protective immunity in the absence of B cells. Immunology 96(1):22-28

Andresen K, Tom TD, Strand M (1991) Characterization of cDNA clones encoding a novel calcium-activated neutral proteinase from Schistosoma mansoni. J Biol Chem 266(23):15085-15090

Angeles JM et al (2015) Water buffalo as sentinel animals for schistosomiasis surveillance. Bull World Health Organ 93(7):511-512. https://doi.org/10.2471/BLT.14.143065

Barry MA, Simon GG, Mistry N, Hotez PJ (2013) Global trends in neglected tropical disease control and elimination: impact on child health. Arch Dis Child 98(8):635-641. https://doi.org/10.1136/ archdischild-2012-302338

Braschi S, Wilson RA (2006) Proteins exposed at the adult schistosome surface revealed by biotinylation. Mol Cell Proteomics 5(2):347356. https://doi.org/10.1074/mcp.M500287-MCP200 
Byram JE, Doenhoff MJ, Musallam R, Brink LH, von Lichtenberg F (1979) Schistosoma mansoni infections in T-cell deprived mice, and the ameliorating effect of administering homologous chronic infection serum. II. Pathology. Am J Trop Med Hyg 28(2):274-285

Chen D, Luo X, Xie H, Gao Z, Fang H, Huang J (2013) Characteristics of IL-17 induction by Schistosoma japonicum infection in C57BL/6 mouse liver. Immunology 139(4):523-532. https://doi.org/10.1111/ imm. 12105

Coler RN et al (2011) Development and characterization of synthetic glucopyranosyl lipid adjuvant system as a vaccine adjuvant. PLoS One 6(1):e16333. https://doi.org/10.1371/journal.pone.0016333

Colley DG, Secor WE (2014) Immunology of human schistosomiasis. Parasite Immunol 36(8):347-357. https://doi.org/10.1111/pim. 12087

Colley DG, Bustinduy AL, Secor WE, King CH (2014) Human schistosomiasis. Lancet 383(9936):2253-2264. https://doi.org/10.1016/ S0140-6736(13)61949-2

Dale CJ et al (2006) Prime-boost strategies in DNA vaccines. Methods Mol Med 127:171-197. https://doi.org/10.1385/1-59745-168-1:171

De Rose R et al (2006) Dose-response relationship of DNA and recombinant fowlpox virus prime-boost HIV vaccines: implications for future trials. Hum Vaccin 2(3):134-136

Doenhoff M, Musallam R, Bain J, McGregor A (1979) Schistosoma mansoni infections in T-cell deprived mice, and the ameliorating effect of administering homologous chronic infection serum. I. Pathogenesis. Am J Trop Med Hyg 28(2):260-263

El Ridi R, Tallima H (2013) Vaccine-induced protection against murine schistosomiasis mansoni with larval excretory-secretory antigens and papain or type-2 cytokines. J Parasitol 99(2):194-202. https:// doi.org/10.1645/GE-3186.1

El Ridi R, Othman AA, McManus DP (2015) Editorial: The schistosomiasis vaccine - it is time to stand up. Front Immunol 6:390. https:// doi.org/10.3389/fimmu.2015.00390

El-Khoby T et al (2000) The epidemiology of schistosomiasis in Egypt: summary findings in nine governorates. Am J Trop Med Hyg 62(2 Suppl):88-99

Fonseca CT, Oliveira SC, Alves CC (2015) Eliminating schistosomes through vaccination: what are the best immune weapons? Front Immunol 6:95. https://doi.org/10.3389/fimmu.2015.00095

Frey A, Di Canzio J, Zurakowski D (1998) A statistically defined endpoint titer determination method for immunoassays. J Immunol Methods 221(1-2):35-41

Ganley-Leal LM et al (2005) Comparison of Schistosoma mansoni irradiated cercariae and Sm23 DNA vaccines. Parasite Immunol 27(9): 341-349. https://doi.org/10.1111/j.1365-3024.2005.00785.x

Garcon N, Van Mechelen M (2011) Recent clinical experience with vaccines using MPL- and QS-21-containing adjuvant systems. Expert Rev Vaccines 10(4):471-486. https://doi.org/10.1586/erv.11.29

Ghandour AM (1978) The development of Schistosoma haematobium in the hamster. Ann Trop Med Parasitol 72(3):219-225

Gordon CA et al (2012) High prevalence of Schistosoma japonicum infection in carabao from Samar Province, the Philippines: implications for transmission and control. PLoS Negl Trop Dis 6(9):e1778. https://doi.org/10.1371/journal.pntd.0001778

Gryseels B, Polman K, Clerinx J, Kestens L (2006) Human schistosomiasis. Lancet 368(9541):1106-1118. https://doi.org/10.1016/S01406736(06)69440-3

Honeycutt J, Hammam O, CL F, Hsieh MH (2014) Controversies and challenges in research on urogenital schistosomiasis-associated bladder cancer. Trends Parasitol 30(7):324-332. https://doi.org/10. 1016/j.pt.2014.05.004

Hotez PJ (2009) Mass drug administration and integrated control for the world's high-prevalence neglected tropical diseases. Clin Pharmacol Ther 85(6):659-664. https://doi.org/10.1038/clpt.2009.16

Hotez PJ et al (2014) The global burden of disease study 2010: interpretation and implications for the neglected tropical diseases. PLoS
Negl Trop Dis 8(7):e2865. https://doi.org/10.1371/journal.pntd. 0002865

Jankovic D et al (1996) Calpain is the target antigen of a Th1 clone that transfers protective immunity against Schistosoma mansoni. J Immunol 157(2):806-814

Karmakar S et al (2014a) Cross-species protection: Schistosoma mansoni Sm-p80 vaccine confers protection against Schistosoma haematobium in hamsters and baboons. Vaccine 32(11):12961303. https://doi.org/10.1016/j.vaccine.2013.12.057

Karmakar S et al (2014b) Use of an Sm-p80-based therapeutic vaccine to kill established adult schistosome parasites in chronically infected baboons. J Infect Dis 209(12):1929-1940. https://doi.org/10.1093/ infdis/jiu031

Knopf PM, Nutman TB, Reasoner JA (1977) Schistosoma mansoni: resistance to reinfection in the rat. Exp Parasitol 41(1):74-82

Kumagai T et al (2005) Schistosoma japonicum: localization of calpain in the penetration glands and secretions of cercariae. Exp Parasitol 109(1):53-57. https://doi.org/10.1016/j.exppara.2004.11.001

Le L, Zhang W, Karmakar S, Ahmad G, Torben W, Siddiqui AA (2014) Simultaneous priming with DNA encoding Sm-p80 and boosting with $\mathrm{Sm}-\mathrm{p} 80$ protein confers protection against challenge infection with Schistosoma mansoni in mice. Parasitol Res 113(3):11951200. https://doi.org/10.1007/s00436-014-3757-4

Mangold BL, Dean DA (1986) Passive transfer with serum and IgG antibodies of irradiated cercaria-induced resistance against Schistosoma mansoni in mice. J Immunol 136(7):2644-2648

Martin RM, Brady JL, Lew AM (1998) The need for IgG2c specific antiserum when isotyping antibodies from C57BL/6 and NOD mice. J Immunol Methods 212(2):187-192

McManus DP, Loukas A (2008) Current status of vaccines for schistosomiasis. Clin Microbiol Rev 21(1):225-242. https://doi.org/10.1128/ CMR.00046-07

McManus DP et al (2010) Schistosomiasis in the People's Republic of China: the era of the Three Gorges Dam. Clin Microbiol Rev 23(2): 442-466. https://doi.org/10.1128/CMR.00044-09

Melo TT, Sena IC, Araujo N, Fonseca CT (2014) Antibodies are involved in the protective immunity induced in mice by Schistosoma mansoni schistosomula tegument (Smteg) immunization. Parasite Immunol 36(2):107-111

Mo AX, Agosti JM, Walson JL, Hall BF, Gordon L (2014) Schistosomiasis elimination strategies and potential role of a vaccine in achieving global health goals. Am J Trop Med Hyg 90(1):54-60. https://doi.org/10.4269/ajtmh.13-0467

Molehin AJ, Rojo JU, Siddiqui SZ, Gray SA, Carter D, Siddiqui AA (2016) Development of a schistosomiasis vaccine. Expert Rev Vaccines: 1-9. https://doi.org/10.1586/14760584.2016.1131127

Mosmann TR, Coffman RL (1989) TH1 and TH2 cells: different patterns of lymphokine secretion lead to different functional properties. Annu Rev Immunol 7:145-173. https://doi.org/10.1146/annurev.iy. 07.040189 .001045

Ohta N, Kumagai T, Maruyama H, Yoshida A, He Y, Zhang R (2004) Research on calpain of Schistosoma japonicum as a vaccine candidate. Parasitol Int 53(2):175-181. https://doi.org/10.1016/j.parint. 2004.01.007

Osada Y et al (2001) Protective immunity to Schistosoma japonicum infection depends on the balance of $\mathrm{T}$ helper cytokine responses in mice vaccinated with gamma-irradiated cercariae. Parasite Immunol 23(5):251-258

Pearce EJ, MacDonald AS (2002) The immunobiology of schistosomiasis. Nat Rev Immunol 2(7):499-511. https://doi.org/10.1038/nri843

Pearson MS et al (2012) Enhanced protective efficacy of a chimeric form of the schistosomiasis vaccine antigen Sm-TSP-2. PLoS Negl Trop Dis 6(3):e1564. https://doi.org/10.1371/journal.pntd.0001564

Ricciardi A, Visitsunthorn K, Dalton JP, Ndao M (2016) A vaccine consisting of Schistosoma mansoni cathepsin B formulated in Montanide ISA 720 VG induces high level protection against 
murine schistosomiasis. BMC Infect Dis 16:112. https://oi.org/10. 1186/s12879-016-1444-Z

Rinaldi G, Young ND, Honeycutt JD, Brindley PJ, Gasser RB, Hsieh MH (2015) New research tools for urogenital schistosomiasis. J Infect Dis 211(6):861-869. https://doi.org/10.1093/infdis/jiu527

Schneider J et al (2001) A prime-boost immunisation regimen using DNA followed by recombinant modified vaccinia virus Ankara induces strong cellular immune responses against the Plasmodium falciparum TRAP antigen in chimpanzees. Vaccine 19(32):45954602

Sher A, Hieny S, James SL, Asofsky R (1982) Mechanisms of protective immunity against Schistosoma mansoni infection in mice vaccinated with irradiated cercariae. II. Analysis of immunity in hosts deficient in T lymphocytes, B lymphocytes, or complement. J Immunol 128(4): $1880-1884$

Siddiqui AA et al (1993) Characterization of $\mathrm{Ca}(2+)$-dependent neutral protease (calpain) from human blood flukes, Schistosoma mansoni. Biochim Biophys Acta 1181(1):37-44

Street M et al (1999) TNF is essential for the cell-mediated protective immunity induced by the radiation-attenuated schistosome vaccine. J Immunol 163(8):4489-4494

Tanghe A, Content J, Van Vooren JP, Portaels F, Huygen K (2001a) Protective efficacy of a DNA vaccine encoding antigen 85A from Mycobacterium bovis BCG against Buruli ulcer. Infect Immun 69(9):5403-5411

Tanghe A et al (2001b) Improved immunogenicity and protective efficacy of a tuberculosis DNA vaccine encoding $\mathrm{Ag} 85$ by protein boosting. Infect Immun 69(5):3041-3047. https://doi.org/10.1128/IAI.69.5. 3041-3047.2001

Torben W, Ahmad G, Zhang W, Siddiqui AA (2011) Role of antibodies in Sm-p80-mediated protection against Schistosoma mansoni challenge infection in murine and nonhuman primate models. Vaccine 29(12):2262-2271. https://doi.org/10.1016/j.vaccine.2011.01.040

Torben W et al (2012) Role of antibody dependent cell mediated cytotoxicity (ADCC) in Sm-p80-mediated protection against Schistosoma mansoni. Vaccine 30(48):6753-6758. https://doi.org/10.1016/j. vaccine. 2012.09.026

Tucker MS, Karunaratne LB, Lewis FA, Freitas TC, Liang YS (2013) Schistosomiasis. Curr Protoc Immunol 103:Unit 19 1. https://doi. org/10.1002/0471142735.im1901s103

van der Werf MJ et al (2003) Quantification of clinical morbidity associated with schistosome infection in sub-Saharan Africa. Acta Trop $86(2-3): 125-139$

Varaldo PB et al (2004) Recombinant Mycobacterium bovis BCG expressing the Sm14 antigen of Schistosoma mansoni protects mice from cercarial challenge. Infect Immun 72(6):3336-3343. https:// doi.org/10.1128/IAI.72.6.3336-3343.2004

Viana IR et al (1994) Interferon-gamma production by peripheral blood mononuclear cells from residents of an area endemic for Schistosoma mansoni. Trans R Soc Trop Med Hyg 88(4):466-470

Vuong PN, Bayssade-Dufour C, Albaret JL, Farhati K (1996) Histopathological observations in new and classic models of experimental Schistosoma haematobium infections. Tropical Med Int Health 1(3):348-358

Wang W, Kirschfink M, Ruppel A (2006) Schistosoma japonicum and S. mansoni cercariae: different effects of protein in medium, of mechanical stress, and of an intact complement system on in vitro transformation to schistosomula. Parasitol Res 99(3):269-274. https://doi.org/10.1007/s00436-006-0150-y

Wilson RA, Coulson PS (2009) Immune effector mechanisms against schistosomiasis: looking for a chink in the parasite's armour. Trends Parasitol 25(9):423-431. https://doi.org/10.1016/j.pt.2009. 05.011

Wilson RA, Coulson PS, Mountford AP (1999) Immune responses to the radiation-attenuated schistosome vaccine: what can we learn from knock-out mice? Immunol Lett 65(1-2):117-123

Wilson RA et al (2008) Elimination of Schistosoma mansoni adult worms by rhesus macaques: basis for a therapeutic vaccine? PLoS Negl Trop Dis 2(9):e290. https://doi.org/10.1371/journal.pntd.0000290

Wilson RA, Li XH, Castro-Borges W (2016) Do schistosome vaccine trials in mice have an intrinsic flaw that generates spurious protection data? Parasit Vectors 9(1):89. https://doi.org/10.1186/s13071016-1369-9

Zhang M, Tian F, Gao Y, Ji M, Wu G (2010a) Ultraviolet- attenuated cercariae of Schistosoma japonicum fail to effectively induce a Th1 response in spite of up-regulating expression of cytotoxicity-related genes in C57BL/6 mice. J Biomed Res 24(4):277-284. https://doi. org/10.1016/S1674-8301(10)60039-5

Zhang W et al (2010b) Sm-p80-based DNA vaccine provides baboons with levels of protection against Schistosoma mansoni infection comparable to those achieved by the irradiated cercarial vaccine. J Infect Dis 201(7):1105-1112. https://doi.org/10.1086/651147

Zhang W, Ahmad G, Torben W, Siddiqui AA (2011) Schistosoma mansoni antigen Sm-p80: prophylactic efficacy of a vaccine formulated in human approved plasmid vector and adjuvant (VR 1020 and alum). Acta Trop 118(2):142-151. https://doi.org/10.1016/j. actatropica.2011.01.010

Zhang Y et al (2012) IL-17 neutralization significantly ameliorates hepatic granulomatous inflammation and liver damage in Schistosoma japonicum infected mice. Eur J Immunol 42(6):1523-1535. https://doi.org/10.1002/eji.201141933 